\title{
Sexual behavior of the desert locust during intra- and inter-phase interactions
}

2

4

$5{ }^{1}$ School of Zoology, Tel Aviv University, Tel Aviv, 6997801 Israel and ${ }^{2}$ Department of Entomology,

6 Volcani Center, Bet Dagan 50250, Israel

7

8

9

$10 *$ Correspondance: Amir Ayali: ayali@post.tau.ac.il

11

12 Running title: Locust phases and sexual behavior

13 


\section{Abstract}

Mating and reproduction behaviors and strategies are fundamental aspects of an

17 organism's evolutionary and ecological success. In locusts, intra- as well as inter-phase

18 reproductive interactions among gregarious and solitarious locust populations have a

19 major impact on the locust population dynamics. However, practically all previous work on

20 locust sexual behavior has been limited to the gregarious phase. Here we provide a first

21 detailed description of pre-copulatory behavior of solitarious desert locusts. We compare

22 our findings with those of previous reports of pre-copulatory behavior of gregarious

23 locusts, focusing on the behavioral elements that serve in inter-sex signaling and

24 communication. We also studied inter-phase (mixed pairs) reproductive interactions.

25 Solitarious males were found to invest more in pre-copulatory courtship and signaling

26 compared to their gregarious counterparts; and the solitarious females played a

27 comparatively more dominant role in the inter-sex communication. The solitarious females

28 were also less prone to demonstrate the typical rejection-related behavioral patterns

29 displayed by the gregarious females. As a consequence of the particular characteristic

30 behavior of each phase, the most successful among intra- and inter-phase pairs were

31 gregarious males with solitary females. Least successful were solitary males encountered

32 with gregarious females, indicating a strong asymmetry in inter-phase reproductive

33 interactions. We discuss these results in the context of non-random or assortative mating

34 in locust mixed or sympatric solitarious-gregarious populations.

38 Key words: Schistocerca gregaria, Locust density-dependent phase polyphenism,

39 sexual behavior, reproductive phenotype 


\section{Introduction}

41 The desert locust (Schistocerca gregaria) presents a remarkable example of

42 environmentally-induced behavioral plasticity: changes in population density dramatically

43 alter this locust's behavior. The major behavioral characteristic of locusts in the

44 gregarious phase is that of their strong attraction to conspecifics, which translates to

45 active aggregation behavior (Ellis, 1959; Ellis, 1963; Uvarov, 1966). Gregarious locusts

46 are also generally more active, featuring a strong propensity to march in huge bands of

47 hoppers and form high-density swarms of reproductive adults (recent reviews in Ariel \&

48 Ayali, 2015; Cullen et al., 2017). In contrast, solitary-reared locusts (or locusts from

49 solitarious field populations) actively avoid contact with other locusts (Roessingh et al.,

50 1993; Geva et al., 2010), are more sedentary and cryptic in behavior, do not march, and

51 fly less. Phase transformation is a positive-feedback process. The importance of the

52 change in behavior is that it precedes and facilitates all other phase-changes, from

53 biochemistry and physiology to coloration and morphology (Ariel \& Ayali, 2015; Cullen et

54 al., 2017).

55 Similar to other species demonstrating population cycles (or fluctuations), key factors

56 that affect the dynamics of locust populations are those of mating and reproduction

57 strategies and behaviors. Hence, reproductive success is closely tied to the risks of

58 locust outbreaks (Farrow, 1979; Topaz et al., 2012). Acquiring an understanding of these

59 dynamics calls for an initial in-depth knowledge of the mating and reproductive behavior

60 of locusts. Locust courtship, in general, is considered simple and primitive (Loher, 1959;

61 Uvarov, 1966, 1977; Strong \& Amerasinghe, 1977). Males of S. gregaria were reported

62 to be more dominant during sexual-interactions, while females were reported to show

63 no clear courtship behavioral patterns (Norris, 1954; Pener, 1965, 1967; Strong \&

64 Amerasinghe, 1977; Amerasinghe, 1978; Inayatullah et al., 1994; Golov et al., 2018). A 
65 major feature of the female locust sexual behavior was reported to be rejection of the 66 males: including jumping (before and during mounting), kicking, and movements of the

67 abdomen to prevent copulation (Loher, 1959; Strong \& Amerasinghe, 1977; Uvarov,

68 1977; Golov et al., 2018). We recently provided a first comprehensive description of the

69 pre-copulatory behavior of the gregarious desert locust (Golov et al., 2018), presenting a

70 detailed quantification of the sexual behavior of both sexes, with special emphasis on

71 female choice.

72 The above-noted study by Golov et al. (2018), however, as well as most of the above-

73 mentioned earlier work, was limited to locusts in the gregarious phase (but see

74 Inayatullah et al., 1994). In the gregarious phase, the desert locust displays clear sexual

75 dimorphism, in which fully mature males are bright yellow and females beige-brown to

76 yellowish (Norris, 1954; Pener, 1965; Pener \& Simpson, 2009). In accordance with this,

77 it has been assumed that visual cues are a primary means of sexual recognition and

78 pre-copulatory communication in the gregarious locust (Whitman, 1990; Obeng-Ofori et

79 al., 1993; Inayatullah et al., 1994; Ely et al., 2006). Sexual dimorphism is much less

80 pronounced in the solitarious phase of the desert locust. Very limited knowledge is

81 available regarding sexual recognition and communication, as well as courtship and

82 mating behavior of locusts in the solitarious phase. For example, Tanaka et al. (2003)

83 reported that in isolated-reared adult migratory locusts (Locusta migratoria) the pre-

84 copulatory mounting tends to be shorter than that of crowd-reared ones, while the

85 opposite is true for the length of copulation. In the desert locust, as in all true locust

86 species, the fundamental differences in life history between the two phases are expected

87 to result in major differences in courtship and pre-copulatory behavior. Density-

88 dependent phase differences can be predicted in all stages and aspects of the behavior, 
89 from mate finding and mate recognition, through pre-mounting courtship, to mate choice

90 and sexual conflict.

91 A practically unexplored question of interest, as well as of importance for our

92 understanding of locust population dynamics, is that of the existence and nature of inter-

93 phase reproductive interactions. Locust populations of mixed phase or, alternatively,

94 sympatric populations of gregarious and solitarious locusts, can be found during periods

95 of transition, i.e. in recession areas, during periods of increasing populations, or in

96 migration areas during the breakdown of a swarm (e.g. Tanaka et al., 2010). As noted,

97 density-dependent variations are expected in locust reproductive behavior. Alternative

98 reproductive strategies or tactics are accepted to be aimed at increasing the players'

99 relative reproductive success. However, the interactions of some reproductive

100 phenotypes may restrict gene flow within the species (e.g. assortative mating;

101 Andersson \& Iwasa, 1996; Nosil et al., 2005; Rundle \& Nosil, 2005; Ramirez-Romero et

102 al., 2017). In the case of phase-dependent reproductive phenotypes in locusts, this may

103 translate to limited inter-phase pre-reproductive interactions and an overall preference to

104 mate within the phase.

105 In the current work we set out to present a comparative, detailed description of pre-

106 copulatory behavior of male and female desert locusts in the solitarious phase. We

107 further examined how the pronounced phase-dependent differences affect inter-phase

108 encounters and the success of inter-phase copulation. Our findings have important

109 consequences for understanding locust population dynamics, and specifically the

110 dynamics of phase change and the development of a locust outbreak. 


\section{$114 \quad$ Material \& Methods}

115 Animals

116 Desert locusts, Schistocerca gregaria (Forskål 1775), from our colony at Tel Aviv

117 University were reared for many consecutive generations under crowded conditions (i.e.

118 approaching the gregarious phase), 100-160 individuals in 60L aluminum cages. In order

119 to obtain locusts in the solitarious phase, hatchlings from eggs laid by the gregarious

120 females were collected within $2 \mathrm{~h}$ of hatching and reared in isolation in $1 \mathrm{~L}$ cages until

121 adulthood (Geva et al., 2010). Extra care was taken to maintain similar rearing

122 conditions for both gregarious and solitarious locusts, with the exception of density.

123 Cages were located in two dedicated room (separating between gregarious and

124 solitarious colonies) under a constant temperature $\left(29-31^{\circ} \mathrm{C}\right)$ and light cycle of $12 \mathrm{~L}: 12 \mathrm{D}$.

125 Supplementary radiant heat was supplied during day-time by incandescent $25 \mathrm{~W}$ electric

126 bulbs (full visible spectrum, yellow and red dominant), resulting in a day temperature of

127 ca. $37^{\circ} \mathrm{C}$. Locusts were provided daily with fresh wheat and dry oats, and plastic cups

128 filled with moist sand for oviposition.

129 Adult locusts used in all experiments were virgin males and females. Gregarious virgin

130 adults were obtained by marking newly-emerged adults with non-poisonous acrylic paint

131 within 24 hours following ecdysis. Males and females were separated into single- sex

132 "cohort cages" every 3 days. Thus, in each cohort cage the maximum age range of the

133 individual locusts was less than 72 hours. The cages were maintained under the same

134 rearing conditions as above. Based on our preliminary work and previous reports

135 (Injeyan \& Tobe, 1981; Mahamat et al., 1993; Wybrandt \& Andersen, 2001; Ely et al.,

136 2006; Nishide \& Tanaka, 2012), gregarious locusts in our experiments were sexually

137 mature at 12-14-days-old in males (when the yellowish coloration had reached stage $\mathrm{V}$;

138 see Norris, 1954; Loher, 1961), and at 18-20 days-old in females. The maturation period 
139 is longer in solitarious locusts than in their gregarious counterparts (Norris, 1954; Norris,

140 1962; Pener, 1976; Amerasinghe, 1978)). Hence, the age of solitarious experimental

141 animals of both sexes was 30-32 days.

\section{Experimental design}

143 Experiments were carried out as described by Golov et al. (2018). Briefly, a male and a

144 female locust, in the solitary phase, were introduced into the two sides of an observation

145 cell $(14 \times 13 \times 24 \mathrm{~cm}$, divided in two by an opaque plastic partition). Five minutes after

146 introducing the locusts into the cell the experiment was initiated by carefully removing

147 the partition between the compartments, and replacing an initial red light illumination with

148 two regular $25 \mathrm{~W}$ light bulbs. Experiments lasted up to 3 hours, or until copulation had

149 occurred, if earlier, and were recorded by a SONY HDR-PJ820E video camera.

150 Mixed inter-phase experiments examining the interactions between the phases were

151 conducted in a similar manner as the above, with either a solitary female and a

152 gregarious male or a gregarious female and a solitary male introduced into the cage.

\section{Data analyses}

154 The recorded videos of the behavior of each pair were reviewed and analyzed using 155 BORIS software (Friard \& Gamba, 2016). Data analyses mostly followed the detailed 156 description provided by Golov et al. (2018). In brief, behavioral elements were identified

157 in order to describe the locusts' pre-copulatory behavior. These included both repetitive 158 (prolonged, e.g. the vibration of the hind leg femur) and discrete (momentary, e.g.

159 jumping) behaviors. The two behavioral types were counted, with a 'count' relating to the 160 duration of a behavior from initiation until termination. Behavioral measurements were 161 taken only if the male and female were at a distance of less than $10 \mathrm{~cm}$ (depicted as an

162 'encounter'). For both pre-mounting and mounting behavioral elements, the following 163 parameters were measured and compared for both sexes: (1) In order to obtain the 
164 pattern or chronological sequence of the behavioral repertoire, the relative latency to

165 initiation of each behavior was noted (relative to the total time of the relevant stage,

166 either pre-mounting or mounting). (2) The probability of a specific behavior occurring

167 (per experiment, $\mathrm{PO}=1$ if the behavior occurred at least once, and 0 otherwise). (3) The

168 frequency of occurrences of a specific behavioral element.

169 Kinematic diagrams were constructed for all the transitions between pairs of behavioral

170 elements (i.e., preceding-following elements), for males and females, as detailed in

171 (Golov et al., 2018). An average transition probability (TP) was calculated based on all

172 possible transitions between each pair of elements. For values of TP between two

173 elements above $10 \%$, a difference of $15 \%$ or more, in solitary vs. gregarious locusts,

174 was set as a threshold in constructing the comparative transitions diagrams presented.

175 The statistical data analysis and output were conducted in GraphPad Prism version 6.04

176 for Windows, JMP®, Version 12.0.1 SAS Institute, and Canvas draw 2.0 (Deneba

177 Systems, Miami, FL). Comparison were conducted using two-sided Fisher exact test

178 with Bonferroni adjustments as required (for multiple comparisons).

\section{Results}

180 We first set out to obtain an in-depth comparative description of the pre-copulatory

181 behavior of male and female locusts in the solitarious phase ( $\mathrm{N}=26$ pairs). Figure 1

182 presents, in a simple and schematic manner, the consecutive stages (S1-7) leading from

183 first encounter to copulation of a solitarious locust pair, divided into the pre-mounting and

184 the mounting stages. In Supplementary Figure 1 we detail the behavioral elements

185 comprising each stage, as well as the probability of each element to occur (PO),

186 separately for males and females, in a similar manner to that used to describe the

187 gregarious locusts' behavior in Golov et al. (2018) (see Fig.1 therein). 
188 In order to facilitate a comparative investigation of phase-related differences in pre-

189 copulatory behavior, we focused our attention on those behavioral elements that we

190 consider fundamental for inter-sex communication (Figs. 2 and 3; raw data for the

191 gregarious locusts are mostly those found in Golov et al., 2018. N=31; Note that the

192 current study included the behavioral data of all pairs tested, unlike Golov et al., 2018

193 that presented only data from experiments that culminated in successful copulation).

194 Generally, the major phase-related differences were manifested in the pre-mounting

195 stage (cf. Figs 2 and 3 with the different stages noted in Fig 1S). As demonstrated in

196 Figure 2A, solitarious males showed a much higher prevalence of leg vibrations and

197 wing stridulations (see also Figure S2, demonstrating the higher prevalence of

198 transitions to leg and wing signaling in the solitary males). In contrast, a slow repetitive

199 elevation of the hind legs as well as signals associated with the abdomen (abdominal

200 wagging) are behavioral elements much less characteristic of the solitarious males than

201 the gregarious ones. An overall subordinate role of the solitary males in the sexual

202 interactions was manifested by their higher tendency to walk away during pre-mounting

203 (compared to their gregarious counterparts). No phase-related preference was

204 observed for the mode of attempting to mount the female (by way of climbing or jumping;

205 Fig. 2B). Using the wings for signaling (i.e. short and long wing stridulation during pre-

206 mounting) was found to be a characteristic behavior of the solitarious females, almost

207 never demonstrated by gregarious ones (Fig. 3A). A major phase-dependent difference

208 was observed in behavioral elements associated with female rejection, which were much

209 reduced in solitary females (Fig. 3B; Fig S3).

210 Next, we addressed the nature of the inter-phase reproductive interactions by repeating

211 the experiments presented above, this time with "mixed-phase" pairs. Interestingly, we

212 found that the locusts tended to preserve the dominant features of their sexual behavior, 
213 irrespective of the phase-identity of their mate (cf. Figs. 4 \& 5 to Figs. $2 \& 3$,

214 respectively). The solitarious males were still greatly preoccupied with leg vibration and

215 wing stridulation, also when encountering a gregarious female; while the gregarious

216 males still displayed intense slow repetitive elevation of their legs also towards a

217 solitarious female (Fig. 4A). Again, the solitarious females displayed intense wing

218 stridulation, as well as reduced rejection-related behavioral patterns, compared to the

219 gregarious ones (Fig. 5A). Most importantly, the two types of inter-phase pairs differed

220 greatly in the rate of successful copulations (Fig. 6): when paired with a female of the

221 other phase, gregarious males were highly successful; whereas the solitarious males

222 were extremely unsuccessful in inter-phase pairing (9 out of 10 pairs mated successfully

223 compared to only 3 out of 10 , respectively; Fig. 6; data for gregarious-gregarious

224 couples were computed from the raw data in Golov et al., 2018). The low rate of

225 successful copulation of the solitary males when paired with gregarious females is

226 further emphasized when compared with the relatively high success rates (more than

$22780 \%$ ) demonstrated when interacting with solitarious females.

\section{Discussion}

230 Unique, species-specific, sexual behavior (i.e. courtship and mating-related behavioral

231 patterns) is one of the hallmarks of a species, functioning both to defining and maintain

232 species differentiation (Rundle \& Nosil, 2005; Nosil, 2012). Hence, it is expected that

233 courtship and mating-related behavioral patterns will be robust or highly consistent within

234 the sexes of a species. However, ample research has revealed various sources of

235 diversity or variation in sexual behavior and reproductive strategies within a species and,

236 moreover, within sexes (Gross, 1996; Rhen \& Crews, 2002). 
237 Locust density-dependent phase polyphenism offers an extreme example of

238 environmentally-induced plasticity, including very pronounced phase-dependent

239 differences in behavior. The very different social environment of locusts in the gregarious

240 or the solitarious phase is expected to result in pronounced differences in courtship and

241 mating-related behavioral patterns. In a dense swarm of sexually mature gregarious

242 locusts there is fierce competition among males. Field studies of S. gregaria have

243 indicated that males outnumber females at the pairing and oviposition sites, and that

244 there are severe struggles between males for the possession of females (Ellis \& Ashall,

245 1957; Popov, 1958). This results in males displaying a highly active, dominant role in

246 inter-sexual interactions (Golov et al., 2018), with very basic or limited courtship, as also

247 shown for the migratory locust (Oberlin, 1973), and which is assumed to be a

248 characteristic of most Catantopinae (Otte, 1970). Strong et al. (1977) also suggested

249 that limited male courtship is a characteristic of gregarious populations that experience

250 strong intra-male competition. The gregarious females' pre-copulatory behavior is mainly

251 expressed by behavioral patterns aimed at rejecting the males (e.g. jumping and kicking,

252 bending the abdomen: (Popov, 1958; Loher, 1959; Strong \& Amerasinghe, 1977; Golov

253 et al., 2018), as known for most Catantopinae (Otte, 1970; Golov et al., 2018).

254 In marked contrast, the solitarious locusts' life history involves very different and major

255 challenges in the path to successful mating and reproduction. These include: (1) locating

256 a mate: while both male and female solitarious locusts may participate in mate location,

257 it is accepted that it is mainly the males that are faced with the challenge of searching for

258 and locating the females in a scarce population (Ely et al., 2006; Maeno et al., 2016);

259 (2) sex recognition: this is not straightforward, since sexual dimorphism is very limited in

260 the solitarious locusts compared to the gregarious phase (Norris, 1954; Pener, 1967;

261 Pener \& Simpson, 2009). Moreover, the overall behavior of the solitarious phase is 
characterized as cryptic - not supporting sex-related advertising; and (3) last but not

263 least, there is a need to overcome the inherent tendency of solitarious locusts to repulse

264 and avoid contact with conspecifics. These challenges should have led to: (a) the

265 development of mechanisms dedicated to finding a mate. This important, interesting,

266 and very little studied issue (e.g. Ely et al., 2006) is, however, beyond the scope of the

267 current work; (b) solitarious locusts should employ developed courtship behavior and

268 inter-sex communication to serve in the process of sex recognition; and (c) the mating

269 process of the solitarious pair should be enhanced by reinforcment signals serving to

270 overcome their inherent repulsion tendency.

271 Indeed, our current findings well demonstrate the latter two points: Solitarious males

272 were found to engage significantly more in stridulation behaviors (short and long), as

273 well as in hind leg vibration, compared to gregarious males. Both behaviors may have an

274 important role as signaling stimuli in inter-sex communication. Short and long

275 stridulations were also demonstrated significantly more often by the solitarious

276 compared to the gregarious females. Interestingly, these patterns of male courtship prior

277 to mounting are not common among Catantopinae (Otte, 1970). Consistent with our

278 hypothesis of a need for continuous reinforcement of the contact between the male and

279 female, high level stridulation and leg vibration in the solitarious pairs were also

280 extended into the mounting stage.

281 Evolution and sexual selection must have conveyed reproduction-related "benefits" for

282 locusts in the solitarious phase that balance the above-detailed obstacles. These include

283 greatly reduced intra-sex competition, and, as evident from our practically null

284 observations of female rejection, little mating-related inter-sex conflict. It is possible,

285 however, that the balance of obstacles vs. benefits differs for the solitarious desert locust

286 males and females. The limited male-male competition probably leads the solitarious 
287 males to demonstrate limited intra-sex signaling, reduced assertiveness, and a general

288 "low key" pre-copulatory behavior. On the other hand, solitarious females are tolerant, or 289 even highly receptive during pre-mounting courtship, demonstrating very limited

290 rejection-related behaviors throughout. Therefore, in respect to mating and reproduction, 291 solitarious males and females may have different strategies. Such a sex-dependent

292 strategy will have profound effects on the phase-related population dynamics, a point

293 that should be further investigated both experimentally and in theoretical modeling.

294 The current research introduces another very important issue that should be considered

295 in theoretical studies of locust population dynamics, and specifically phase-change 296 dynamics (rarely addressed in theoretical modeling ; Topaz et al., 2012; Ariel \& Ayali, 297 2015). Fundamental differences in pre-copulatory and courtship behavior are known to 298 contribute to species-level reproductive isolation (e.g. Panhuis et al., 2001; Masta \& 299 Maddison, 2002; Gray, 2005). Pre-mating isolation can result in significant levels of 300 reproductive isolation, even in cases in which other reproductive isolation mechanisms 301 are lacking (Nosil, 2012). The results of our experiments with the mixed, interphase 302 pairs, clearly indicate that locusts of both phases tend to retain the basic features of their 303 phase-specific pre-copulatory behavior when encountering a mate of the opposite 304 phase. This point is further reinforced when remembering that we have specifically 305 focused on behavioral patterns assumed to play a role in signaling and communication.

306 Hence, has evolution and sexual selection result in limiting gene flow between sympatric 307 solitarious and gregarious populations? A closer look at our results, again, suggests an 308 asymmetry: i.e. that this may only be relevant to mixed pairs comprising a solitarious 309 male and a gregarious female. Such a pair comprises "a male from Mars and a female 310 from Venus" (in paraphrase of Gray, 1992), i.e. a pair with very different and far from 311 complementary courtship and pre-copulatory behavioral phenotypes, to the extent that 
312 these profound differences may indeed serve to restrict inter-phase mating (as evident

313 by our finding regarding low copulation success rates in such pairs). In marked contrast,

314 the reciprocal pair, i.e. a gregarious male and a solitarious female, present a 'tight

315 match' of two assertive and receptive counterparts, promoting rapid and high mating

316 rates. Inter-phase sexual interactions may, therefore, be a case of asymmetrical sexual

317 isolation (Arthur \& Dyer, 2015). One outcome of the above may be an enhanced

318 recruitment of solitarious females to a newly-formed gregarious group or a swarm

319 passing by in an area inhabited by a solitarious locust population. Under specific

320 conditions this can even constitute a mechanism for increasing the size of the swarm

321 and escalating a locust outbreak.

322 The above findings may also assist in resolving a longstanding puzzle in the locust

323 literature. Small populations of solitarious locusts should be at risk due to in-breeding

324 and little overall gene flow. However, recent reports, investigating solitarious desert

325 locust populations within a very large geographical range, have indicated a relatively

326 high within-population genetic diversity and low between-population genetic structure

327 (Chapuis et al., 2014; Lazar et al., 2016). In contrast, significant genetic differentiation

328 among desert locust recession populations was also reported (Ibrahim et al., 2000),

329 suggesting that meta-population dynamics could produce genetic divergence among

330 highly scattered populations (Ibrahim, 2001). In addition, in the migratory locust, Locusta

331 migratoria, an homogenizing effect of past swarm events on solitarious populations was

332 reported (Chapuis et al., 2009). Our currently reported results, suggesting an additional

333 layer of complexity in phase dynamics related to phase differences in mating and

334 reproduction behavior as well as to intra-phase sex-dependent differential strategies,

335 may aid in resolving such inconsistencies in solitarious desert locust population

336 dynamics. For example, an inclination of solitary females to mate with gregarious males 
will allow a traveling group of gregarious locusts to serve as an instrument of genetic homogenization of remote solitarious populations. These ideas call for further research in the lab as well as in the field.

\section{Acknowledgments}

We are grateful to Moshe Guershon for his assistance in the experiments and analysis and for live discussions. This work was funded by a grant from the Israel Ministry of Agriculture and Rural Development (891-0277-13). The work in its final stage was partially supported by the German Research Council (DFG; Grant RI 2728/2-1).

\section{Bibliography}

Amerasinghe, F.P. (1978) Effects of J.H.I and J.H.III on yellowing, sexual activity and pheromone production in allatectomized male Schistocerca gregaria. Journal of Insect Physiology, 24, 603-611.

Andersson, M. \& Iwasa, Y. (1996) Sexual selection. Trends in Ecology \& Evolution, 11, 53-58.

Ariel, G. \& Ayali, A. (2015) Locust collective motion and its modeling. PLoS Computational Biology, 11, e1004522.

Arthur, N.J. \& Dyer, K.A. (2015) Asymmetrical sexual isolation but no postmating isolation between the closely related species Drosophila suboccidentalis and Drosophila occidentalis. BMC Evolutionary Biology, 15, 38.

Chapuis, M.-P., Loiseau, A., Michalakis, Y., Lecoq, M., Franc, A. \& Estoup, A. (2009) Outbreaks, gene flow and effective population size in the migratory locust, Locusta migratoria: a regional-scale comparative survey. Molecular Ecology, 18, 792-800.

Chapuis, M.-P., Plantamp, C., Blondin, L., Pagès, C., Vassal, J.-M. \& Lecoq, M. (2014) Demographic processes shaping genetic variation of the solitarious phase of the desert locust. Molecular Ecology, 23, 1749-1763.

Cullen, D.A., Cease, A.J., Latchininsky, A.V., Ayali, A., Berry, K., Buhl, J., et al. (2017) From molecules to management: mechanisms and consequences of locust phase polyphenism. In Advances in Insect Physiology. Academic Press, pp. 167-285.

Ellis, P.E. (1959) Learning and social aggregation in locust hoppers. Animal Behaviour, 7, 91-IN4. 
Ellis, P.E. (1963) Changes in the social aggregation of locust hoppers with changes in rearing conditions. Animal Behaviour, 11, 152-160.

372 Ellis, P.E. \& Ashall, C. (1957) Field Studies on diurnal Behaviour, Movement and 373 Aggregation in the Desert Locust (Schistocerca gregaria Forskăl). Field Studies on 374 diurnal Behaviour, Movement and Aggregation in the Desert Locust (Schistocerca 375 gregaria Forskál).

376 Ely, S.O., Mahamat, H., Njagi, P.G.N., Bashir, M.O., El-Amin, S.E.-T. \& Hassanali, A. (2006) Mate location mechanism and phase-related mate preferences in solitarius desert locust, Schistocerca gregaria. Journal of Chemical Ecology, 32, 1057-1069.

Farrow, R.A. (1979) Population dynamics of the australian plague locust, chortoicetes terminifera (walker), in central western new south wales. I. reproduction and migration in relation to weather. Australian journal of zoology, 27, 717.

382 Friard, O. \& Gamba, M. (2016) BORIS: a free, versatile open-source event-logging 383 software for video/audio coding and live observations. Methods in ecology and evolution 384 / British Ecological Society, 7, 1325-1330.

385 Geva, N., Guershon, M., Orlova, M. \& Ayali, A. (2010) Memoirs of a locust: density386 dependent behavioral change as a model for learning and memory. Neurobiology of 387 Learning and Memory, 93, 175-182.

388 Golov, Y., Rillich, J., Harari, A. \& Ayali, A. (2018) Precopulatory behavior and sexual 389 conflict in the desert locust. PeerJ, 6, e4356.

390 Gray, D.A. (2005) Does courtship behavior contribute to species-level reproductive 391 isolation in field crickets? Behavioral Ecology, 16, 201-206.

392 Gray, J. (1992) Men are from Mars, women are from Venus: A practical guide for 393 improving communication and getting what you want in your relationships. 1st ed. 394 HarperCollins, New York, NY.

395 Gross, M.R. (1996) Alternative reproductive strategies and tactics: diversity within sexes. 396 Trends in Ecology \& Evolution, 11, 92-98. Ibrahim, K.M. (2001) Plague dynamics and population genetics of the desert locust: can turnover during recession maintain population genetic structure? Molecular Ecology, 10, 581-591.

Ibrahim, K.M., Sourrouille, P. \& Hewitt, G.M. (2000) Are recession populations of the desert locust (Schistocerca gregaria) remnants of past swarms? Molecular Ecology, 9,

403 Inayatullah, C., El Bashir, S. \& Hassanali, A. (1994) Sexual Behavior and

404 Communication in the Desert Locust, Schistocerca gregaria (Orthoptera: Acrididae): Sex 405 Pheromone in Solitaria. Environmental Entomology, 23, 1544-1551.

406 Injeyan, H.S. \& Tobe, S.S. (1981) Phase polymorphism in Schistocerca gregaria: 407 Reproductive parameters. Journal of Insect Physiology, 27, 97-102. 
408

Lazar, M., Piou, C., Doumandji-Mitiche, B. \& Lecoq, M. (2016) Importance of solitarious desert locust population dynamics: lessons from historical survey data in Algeria. Entomologia experimentalis et applicata, 161, 168-180.

Loher, W. (1959) Contributions to the study of the sexual behaviour of schistocerca gregaria forskål (orthoptera: acrididae). Proceedings of the Royal Entomological Society of London. Series A, General Entomology, 34, 49-56.

Loher, W. (1961) The chemical acceleration of the maturation process and its hormonal control in the male of the desert locust. Proceedings of the Royal Society B: Biological Sciences, 153, 380-397.

Maeno, K.O., Ould Ely, S., Nakamura, S., Abdellaoui, K., Cissé, S., Jaavar, M.E.H., et al. (2016) Daily microhabitat shifting of solitarious-phase Desert locust adults: implications for meaningful population monitoring. SpringerPlus, 5, 107.

Mahamat, H., Hassanali, A., Odongo, H., Torto, B. \& El-Bashir, E.-S. (1993) Studies on the maturation-accelerating pheromone of the desert locustSchistocerca gregaria (Orthoptera: Acrididae). Chemoecology, 4, 159-164.

Masta, S.E. \& Maddison, W.P. (2002) Sexual selection driving diversification in jumping spiders. Proceedings of the National Academy of Sciences of the United States of America, 99, 4442-4447.

Nishide, Y. \& Tanaka, S. (2012) Yellowing, morphology and behaviour in sexually mature gynandromorphs of the desert locustSchistocerca gregaria. Physiological entomology, 37, 379-383.

Norris, M.J. (1954) Sexual maturation in the desert locust (Schistocerca gregariaForskål) with special reference to the effects of grouping. Anti-Locust Bulletin, 18, 1-44.

Norris, M.J. (1962) Group effects on the activity and behaviour of adult males of the desert locust (Schistocerca gregaria Forsk.) in relation to sexual maturation. Animal Behaviour, 10, 275-291.

Nosil, P. (2012) Ecological Speciation. OUP Oxford, Oxford.

Nosil, P., Vines, T.H. \& Funk, D.J. (2005) Reproductive isolation caused by natural selection against immigrants from divergent habitats . Evolution, 59, 705-719.

Obeng-Ofori, D., Torto, B. \& Hassanali, A. (1993) Evidence for mediation of two releaser pheromones in the aggregation behavior of the gregarious desert locust,Schistocerca gregaria (forskal) (Orthoptera: Acrididae). Journal of Chemical Ecology, 19, 1665-1676.

Oberlin, U.P. (1973) Verhaltensbiologische Studien an der europaeischen Wanderheuschrecke Locusta Migratoria L. Entomol Gesell Basel, 23, 12-23.

Otte, D. (1970) A comparative study of communicative behaviour in grasshoppers. Misc Publ Mus Zool Univ Mich, 141, 1-168.

Panhuis, T.M., Butlin, R., Zuk, M. \& Tregenza, T. (2001) Sexual selection and speciation. Trends in Ecology \& Evolution, 16, 364-371. 
Pener, M.P. (1965) On the influence of corpora allata on maturation and sexual behaviour of Schistocerca gregaria. Journal of zoology, 147, 119-136.

448 Pener, M.P. (1967) Effects of allatectomy and sectioning of the nerves of the corpora allata on oöcyte growth, male sexual behaviour, and colour change in adults of Schistocerca gregaria. Journal of Insect Physiology, 13, 665-684.

Pener, M.P. (1976) The differential effect of the corpora allata on male sexual behaviour in crowded and isolated \{ILocusta migratoria migratorioides\} (R \& F.) males. Acrida, 5, 189-206.

454 Pener, M.P. \& Simpson, S.J. (2009) Locust phase polyphenism: an update. In Advances in insect physiology volume 36, Advances in insect physiology. Elsevier, pp. 1-272. (Schistocerca gregaria Forskal) in eastern Africa. Ecological Studies on Oviposition by Swarms of the Desert Locust (Schistocerca gregaria Forskal) in eastern Africa., 1-70.

Ramirez-Romero, R., Garibay-Benítez, D., Vargas-Ponce, O., Joyce, A. \& Bernal, J.S. (2017) Do assortative mating and immigrant inviability help maintain population genetic structuring of an herbivore on a crop and a wild relative? Insect science (Online).

Rhen, T. \& Crews, D. (2002) Variation in reproductive behaviour within a sex: neural

Roessingh, P., Simpson, S.J. \& James, S. (1993) Analysis of Phase-Related Changes in Sciences, 252, 43-49.

467 Rundle, H.D. \& Nosil, P. (2005) Ecological speciation. Ecology Letters, 8, 336-352.

468 Strong, L. \& Amerasinghe, F.P. (1977) Allatectomy and sexual receptivity in females of 469 Schistocerca gregaria. Journal of Insect Physiology, 23, 131-135.

470 Tanaka, S., Maeno, K., Ould Mohamed, S., Ould Ely, S. \& Babah Ebbe, M.A. (2010)

471 Upsurges of desert locust populations in Mauritania: Body coloration, behavior and

472 morphological characteristics. Applied entomology and zoology, 45, 641-652.

473 Tanaka, S. \& Zhu, D.-H. (2003) Phase-Related Differences in Mating Strategy of a 474 Locust (Orthoptera: Acrididae). Acta Neophilologica, 96, 498-502.

475 Topaz, C.M., D’Orsogna, M.R., Edelstein-Keshet, L. \& Bernoff, A.J. (2012) Locust 476 dynamics: behavioral phase change and swarming. PLoS Computational Biology, 8, 477 e1002642.

478 Uvarov, B. (1977) Grasshoppers and locusts. A handbook of general acridology Vol. 2. 479 Behaviour, ecology, biogeography, population dynamics. cabdirect.org.

480 Uvarov, B.P. (1966) Phase polymorphism. In Grasshoppers and locusts. Cambridge 481 university press, Cambridge, pp. 332-386.

482 Whitman, D.W. (1990) Grasshopper chemical communication. In Biology of

483 Grasshoppers (ed. Chapman, R.F. \& Joern, A.). Wiley, New York, pp. 357-391. 
484 Wybrandt, G.B. \& Andersen, S.O. (2001) Purification and sequence determination of a 485 yellow protein from sexually mature males of the desert locust, Schistocerca gregaria. 486 Insect Biochemistry and Molecular Biology, 31, 1183-1189.

\section{Figure legends:}

491 Figure 1:

492 A simplified schematic description of the consecutive stages (S1-7) comprising the 493 solitarious locusts' pre-copulatory behavioral sequence, from first encounter to 494 copulation. The different stages comprise the premounting and the mounting phases.

\section{Figure 2:}

497 A. The mean probability of occurrence (PO) of several selected male behavioral 498 elements related to sex communication and signaling during pre-mounting. Different 499 colors indicate the body part involved in signaling (indicated on top of bars). B. The 500 mean probability of occurrence (PO) of two types of mounting attempts. Data for 501 solitarious locusts (cross hatched bars, $n=26$ ) are shown in comparison to those of 502 gregarious locusts (open bars, $n=31$; raw data for the gregarious locusts are those found 503 in Golov et al., 2018). ** Statistical significance is indicated: * $p<0.05$ *** $p<0.001$.

505 Figure 3:

506 A. Comparison of female pre-mounting behavioral elements presented in a similar way

507 to that in Fig. 2A. B. The mean probability of occurrence (PO) of behavioral elements 508 associated with female pre-copulatory rejection behavior. (mount) denotes after 509 mounting, or during the mounting phase. Colors and bars as detailed in Fig 2. 
510 Solitarious, $n=26$; gregarious, $n=31$. Raw data for the gregarious locusts are those found

511 in Golov et al., 2018. Statistical significance: * $p<0.05$ *** $p<0.001$.

513 Figure 4:

514 A. Pre-copulatory behavioral elements related to the sexual behavior of males in mixed-

515 phase pairs (GM-SF, Gregarious male and solitarious female; or SM_GF, solitarious

516 male and gregarious female; $n=10$ for both). Colors and bars as detailed in Fig 2. Raw

517 data for the gregarious locusts are those found in Golov et al., 2018. Statistical

518 significance: * $p<0.05 * \star p<0.01$.

520 Figure 5:

521 A. Pre-copulatory behavioral elements related to sexual behavior of the females in

522 mixed-phase pair (GF-SM, Gregarious female and a solitarious male; or SF_GM,

523 solitarious female and a gregarious male; $n=10$ for both). (mount) denotes after

524 mounting, or during the mounting phase. Colors and bars as detailed in Fig 2. Raw data

525 for the gregarious locusts are those found in Golov et al., 2018. Statistical significance: * $526 p<0.05 * * p<0.01$

528 Figure 6:

529 The probability of successful copulations in intra- and interphase pairs. GM and SM

530 indicate gregarious and solitarious males, respectively; GF and SF indicate gregarious

531 and solitarious females, respectively. Data for gregarious-gregarious couples were

532 computed from the raw data found in Golov et al., 2018. Shaded bars denote data for

533 the mixed/interphase pairs. ${ }^{* \star *} p<0.001$ 
535 Supplementary figure 1:

536 The precopulatory behavioral repertoire of the male (left) and female (right) desert locust

537 in the solitary phase (Mean PO: The probability of an element to occur). The pre-

538 mounting and mounting behavioral elements are listed from step 1 to 7 (S1-S7) and

539 color coded according to relevant body part. Behavioral elements that are shared and

540 mutually exhibited by both sexes are presented in italic bold font.

542 Supplementary figure 2:

543 A comparison of the kinematics of the pre-copulatory behavior of solitary and gregarious

544 male locusts; based on a matrix constructed for all transition probabilities (TP) between

545 behavioral elements in each phase (as detailed in the Methods section and following

546 Golov et al., 2018). The color of the circles representing the different behavioral

547 elements corresponds to the color index used in Fig. S1. Arrows represent the direction

548 of a phase-dependent difference of $15 \%$ or more in the TP value between two elements,

549 (red- higher in solitary, black-higher in gregarious locusts).

550

551 Supplementary figure 3 :

552 A comparison of the kinematics of the pre-copulatory behavior of solitary and gregarious 553 female locusts; details as in Fig S2. 


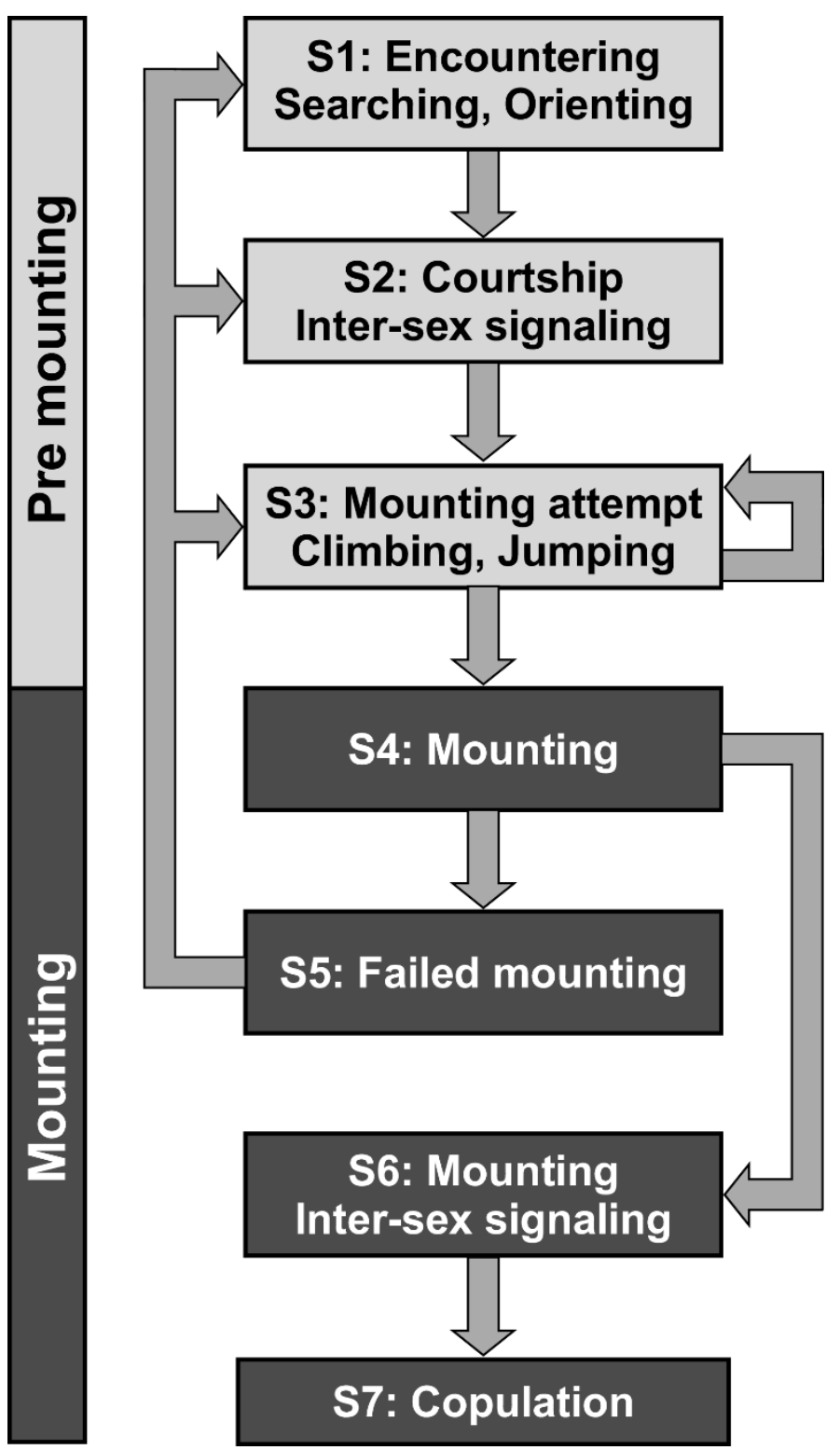

Fig. 1 
A

signal: Hind leg Wing

B Mounting $100]^{* * *} \quad * * * \quad * * * \quad * * * \quad * * * \quad * \quad *$

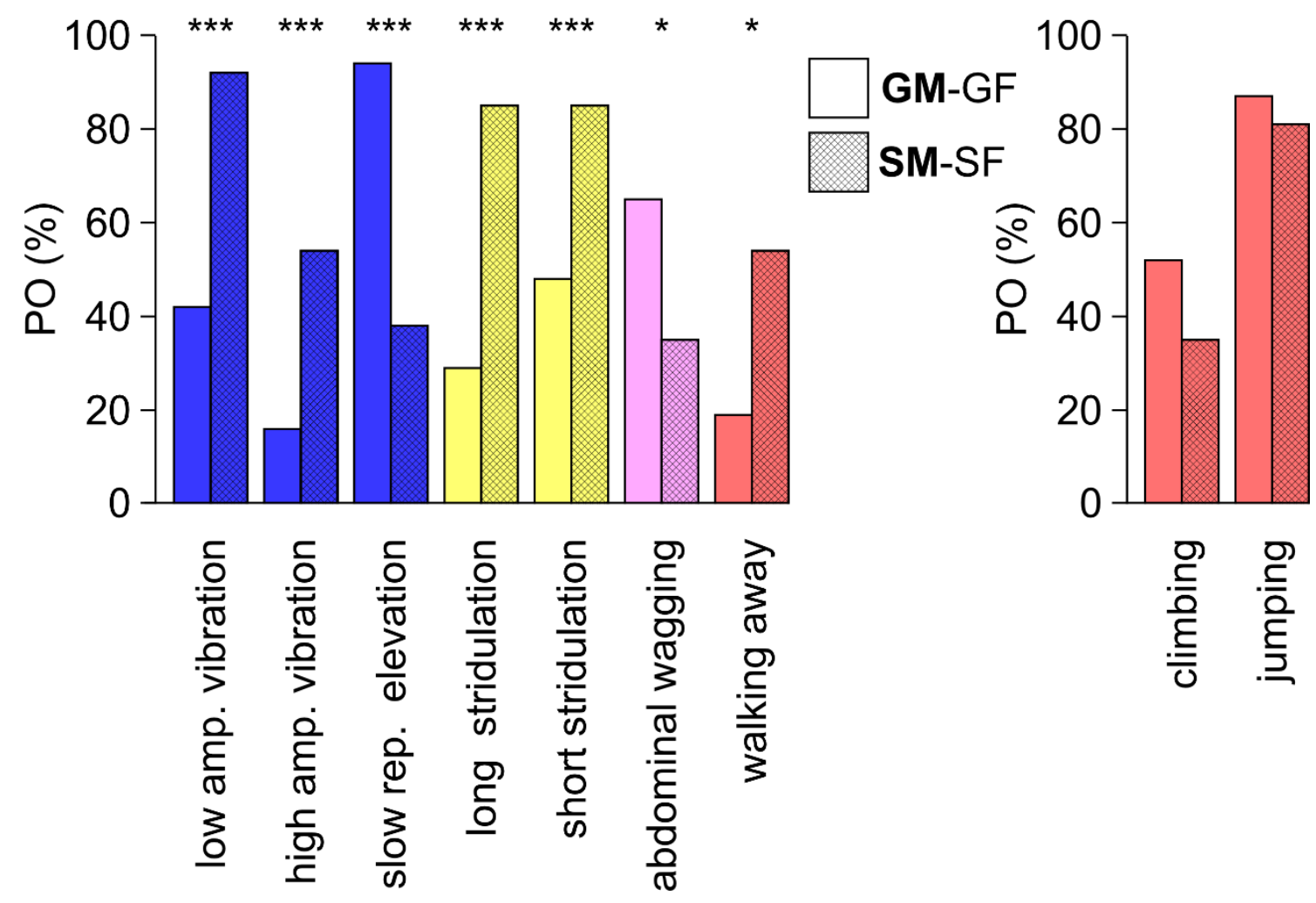

Fig. 2 
A signal: Hind leg Wing Abdomen

B

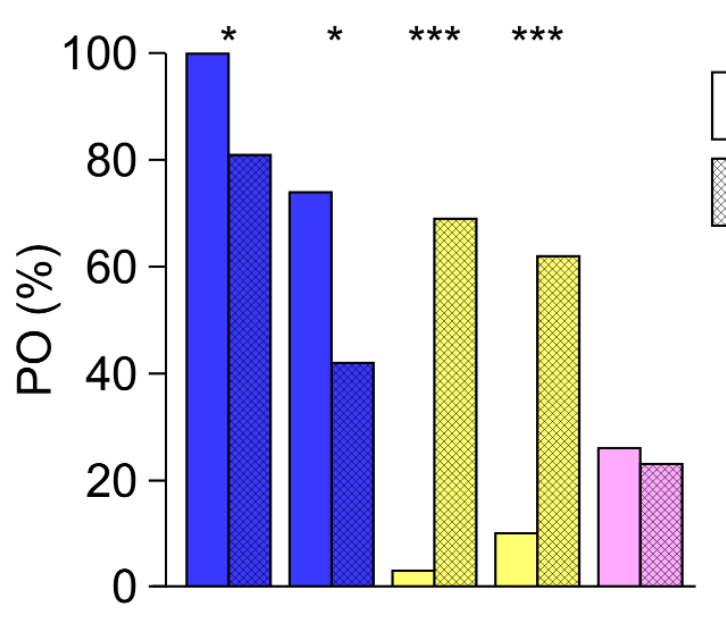

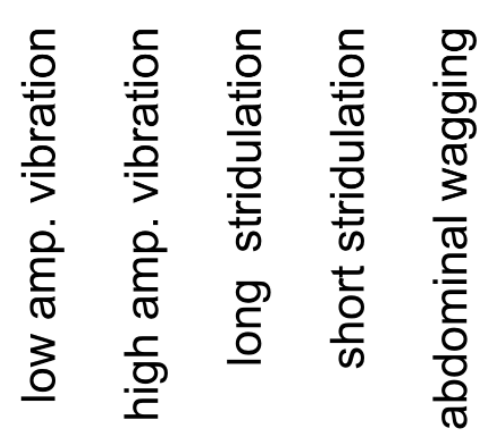

Rejection

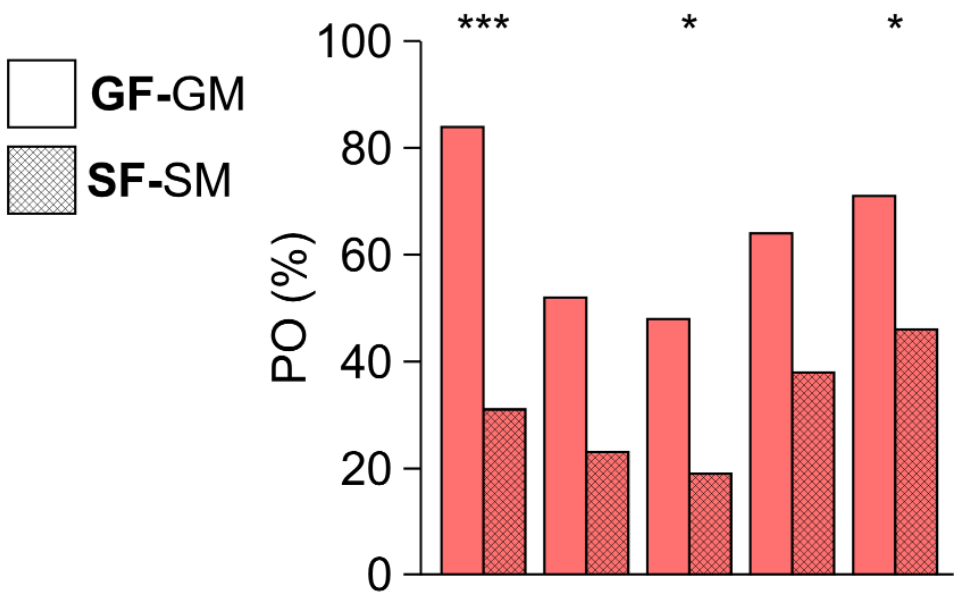

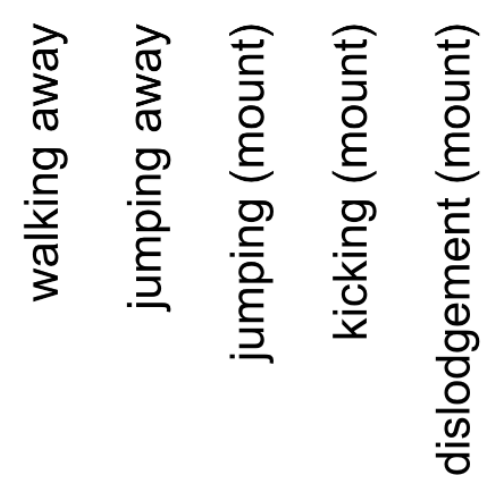

Fig. 3 
A

signal: Hind leg Wing

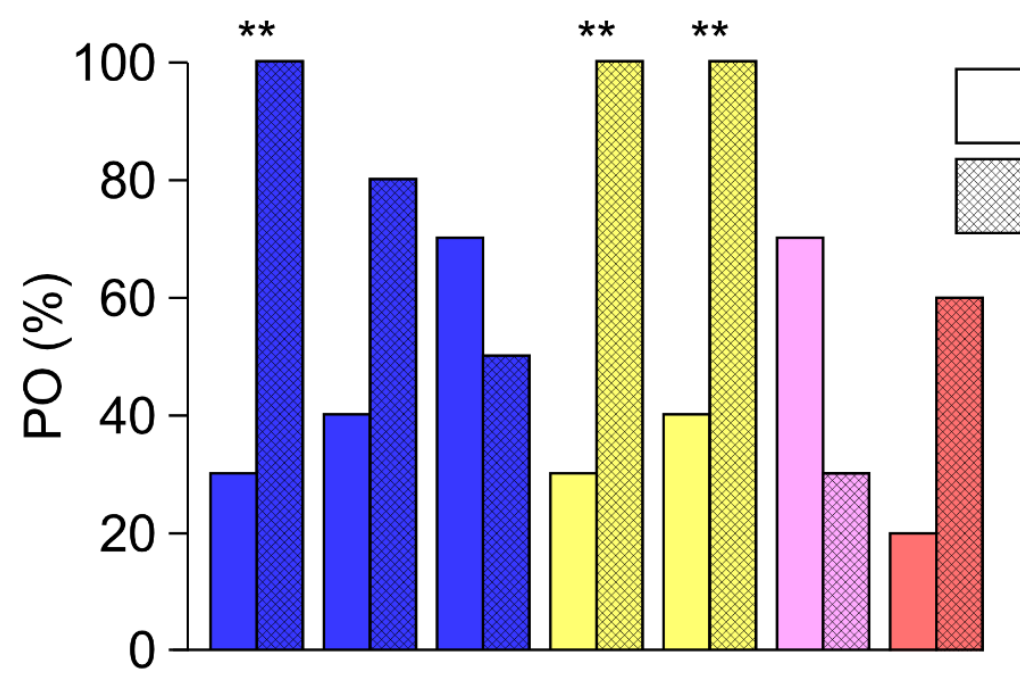

B $\begin{gathered}\text { Mounting } \\ \text { attempt }\end{gathered}$

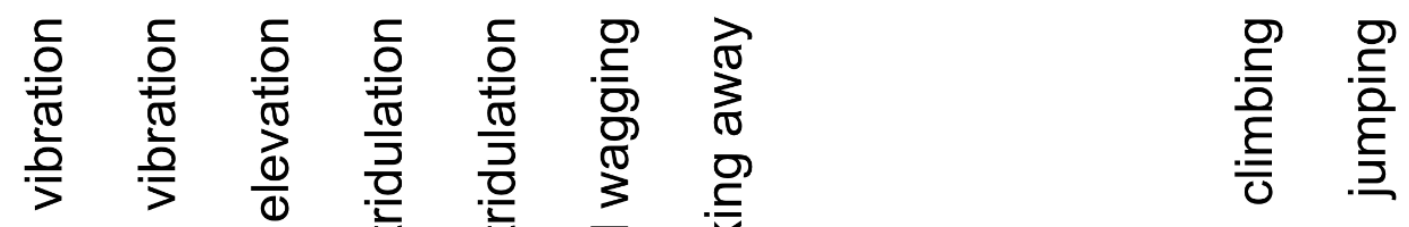

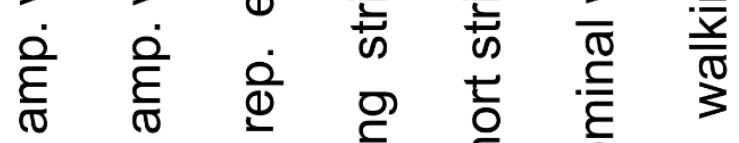

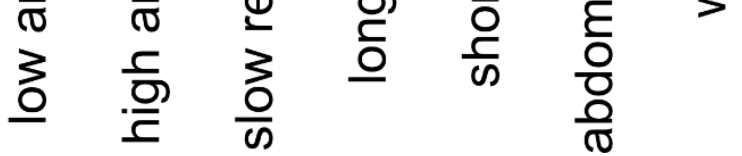

Fig. 4 
A signal: Hind leg Wing Abdomen B
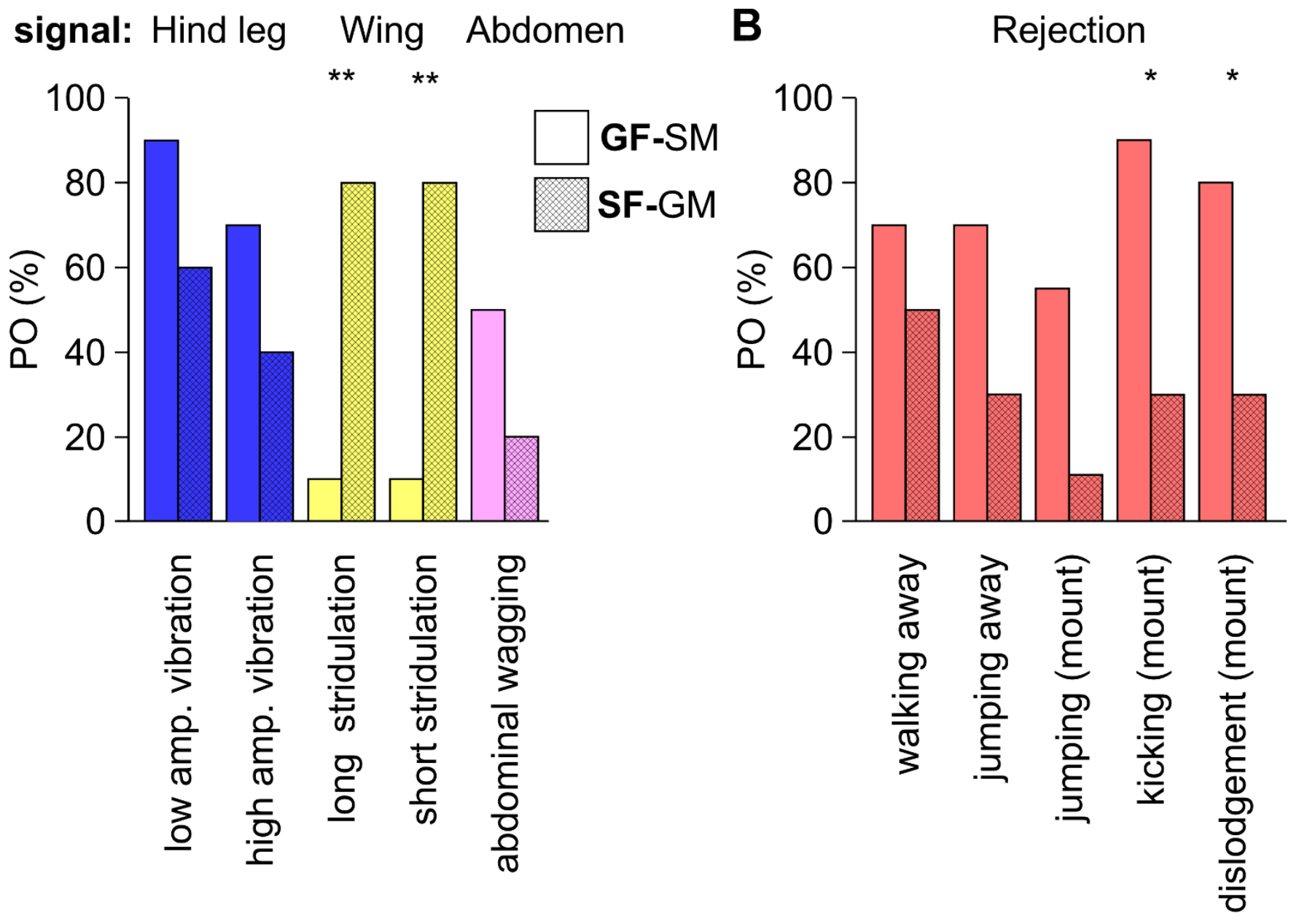

Fig. 5 


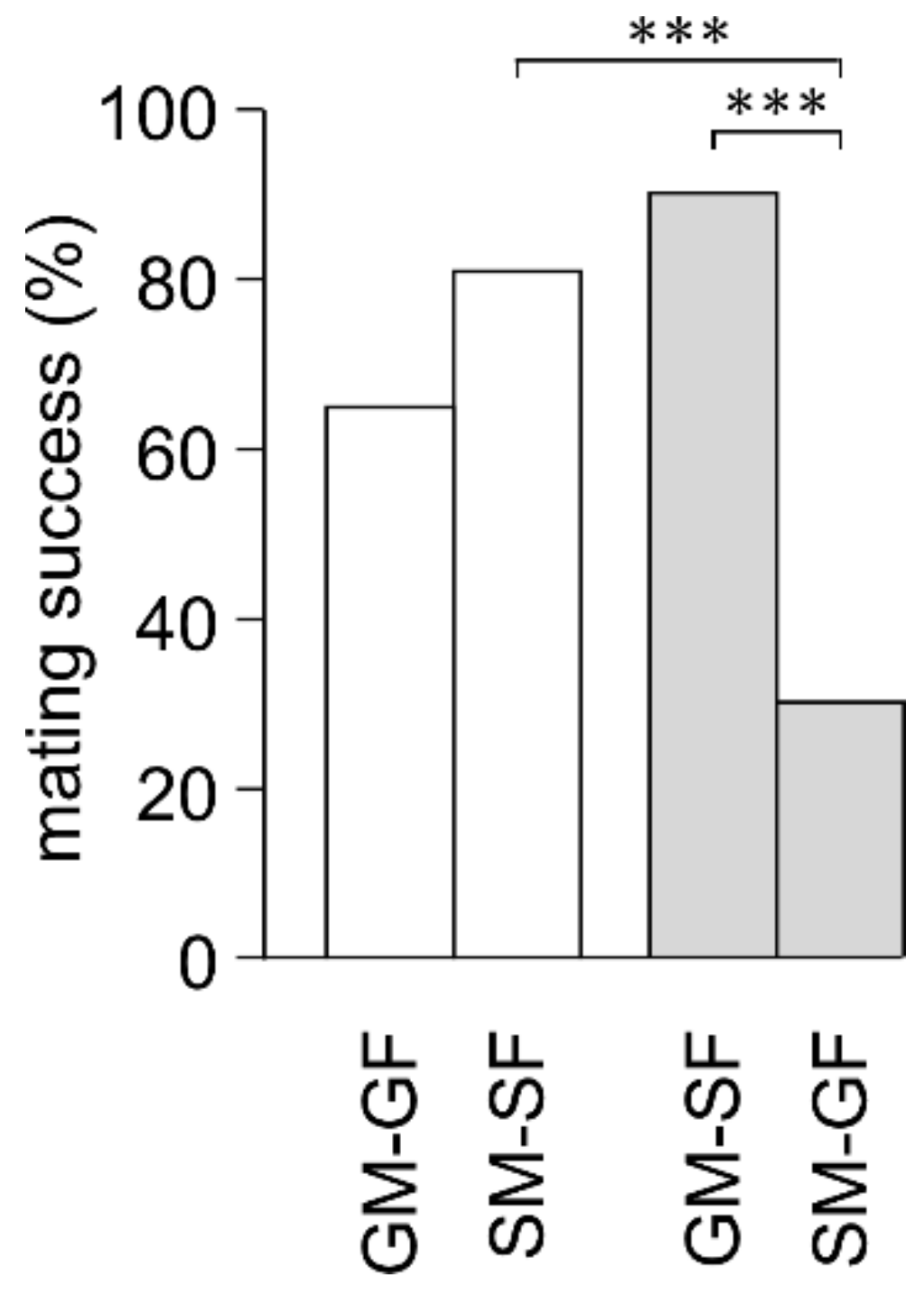

Fig. 6 


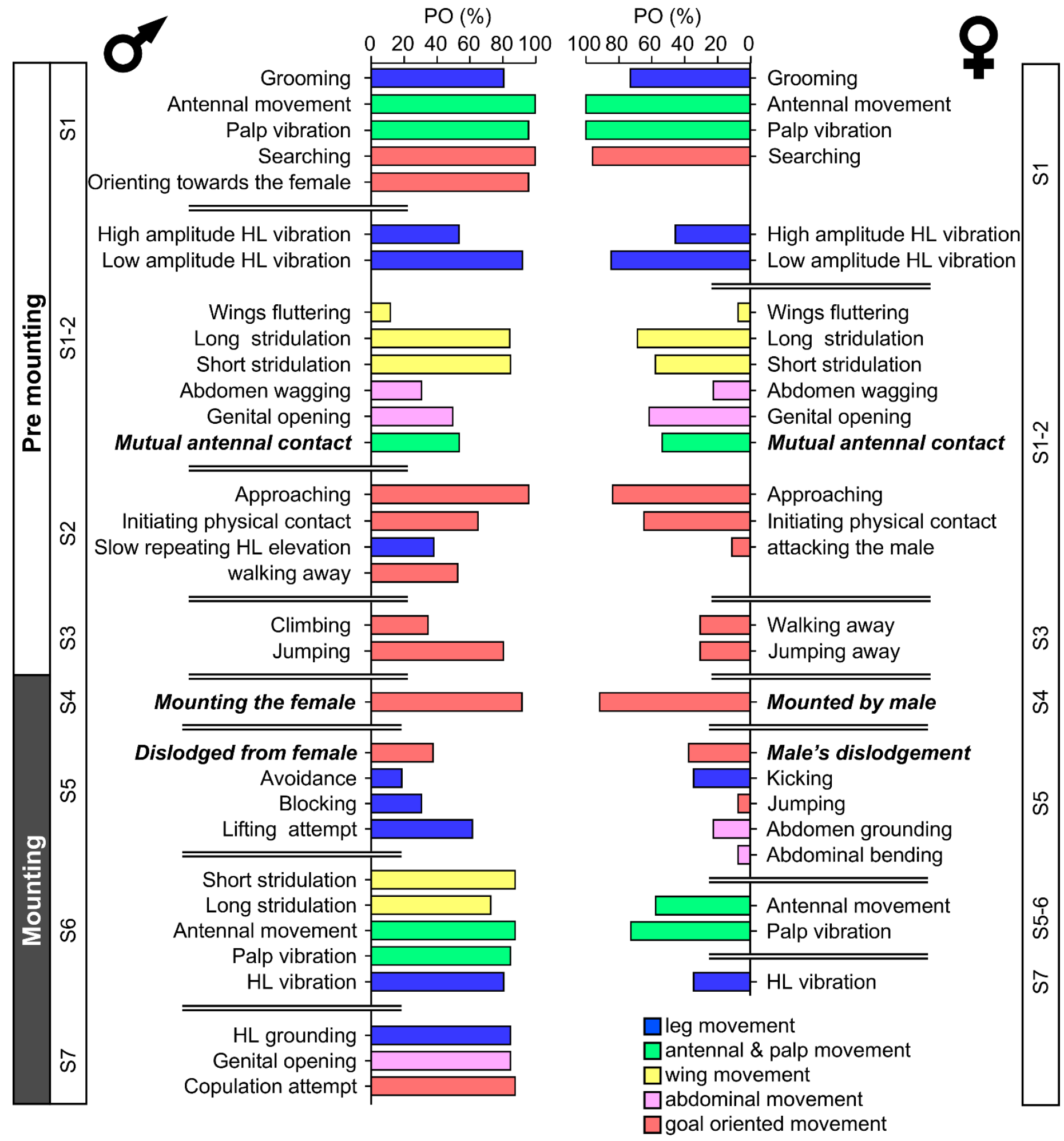




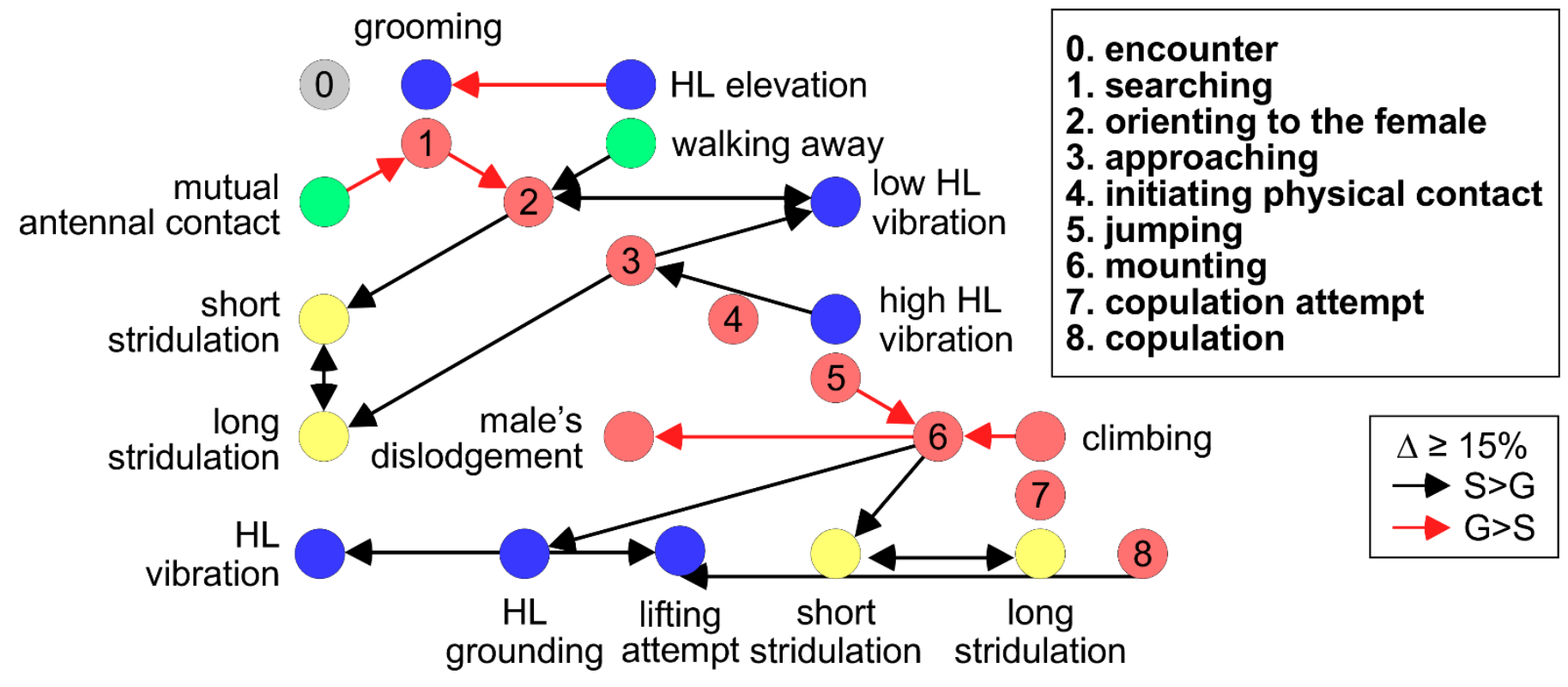

Supp. Fig. 2 


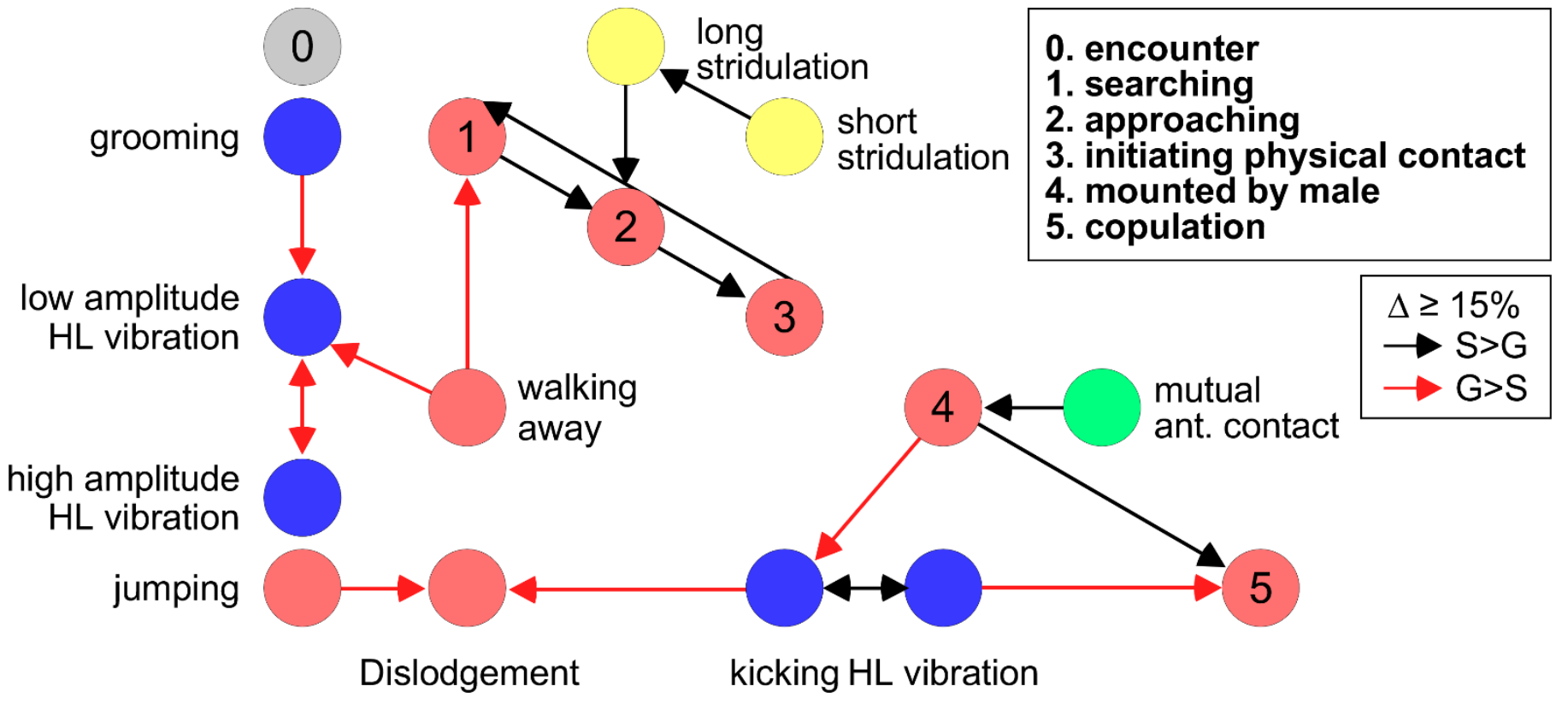

Supp. Fig. 3 